Trabajos y Comunicaciones, 2da. Época, No 48, e064, julio-diciembre 2018. ISSN 2346-8971

Universidad Nacional de La Plata.

Facultad de Humanidades y Ciencias de la Educación.

Departamento de Historia

\title{
Cambios y continuidades en la industria de la alimentación desde un abordaje multiescalar
}

\author{
Mariela Cambiasso * \\ * Universidad Nacional de Buenos Aires - CONICET, Argentina \\ m_cambiasso@hotmail.com
}

Cita sugerida: Cambiasso , M. (2018). Cambios y continuidades en la industria de la alimentación desde un abordaje multiescalar. Trabajos y Comunicaciones (48), e064. https://doi.org/10.24215/23468971e064

Recibido: 5 de abril de 2018 I Aceptado: 21 de mayo de 2018 I Publicado: 27 de julio de 2018

cC)(1) Esta obra está bajo licencia Creative Commons Atribución-NoComercial-CompartirIgual 4.0 Internacional

http://creativecommons.org/licenses/by-nc-sa/4.0/deed.es AR 


\section{Cambios y continuidades en la industria de la alimentación desde un abordaje multiescalar}

Changes and continuities in the food industry from a multiscalar approach

Mariela Cambiasso

Centro de Estudios e Investigaciones Laborales- CONICET

- Universidad Nacional de Buenos Aires, Argentina

m_cambiasso@hotmail.com

\section{Resumen:}

Desde un enfoque cualitativo, el artículo analiza los efectos de la reestructuración capitalista sobre la industria alimenticia de Argentina, centrando la atención en los cambios y continuidades registrados a nivel del sector de actividad, la negociación colectiva y la organización del trabajo en un caso de estudio (Kraft-Mondelez) entre los años noventa y la actualidad. Así, mediante un abordaje multiescalar del problema, busca caracterizarse el escenario en que se desenvuelve la acción sindical y la conflictividad laboral enun sector estratégico de la economía nacional.

Palabras ClaVe: Reestructuración capitalista; Industria alimenticia; Negociación colectiva; Empresa.

\section{Abstract:}

From a qualitative perspective, the article analyzes the effects of capitalist restructuring on Argentina's food industry, focusing attention on the changes and continuities registered at the level of the sector of activity, collective bargaining and the organization of work in a case of study (Kraft-Mondelez) between the nineties and the present. Through a multiscalar approach to the problem, it seeks to characterize the scenario in which union action and labour conflict is developed in a strategic sector of the national economy.

KeYWorDS: Capitalist restructuring; Food industry; Collective bargaining; Company.

\section{INTRODUCCIÓN}

A lo largo del artículo hemos buscado describir la situación de la industria alimenticia local entre los años noventa y la actualidad. Para ello, propusimos un abordaje multiescalar, basado en la articulación de distintos niveles de análisis (el sector de actividad, la negociación colectiva y la empresa), con el objetivo de profundizar la discusión en torno a los cambios y continuidades que podían registrarse.

En el presente trabajo analizamos los efectos de la reestructuración capitalista ${ }^{1}$ sobre la industria de la alimentación en Argentina, centrando la atención en los cambios y continuidades registrados entre los años noventa y la actualidad. El mismo forma parte del recorrido efectuado en mi tesis doctoral, que abordó el estudio de las relaciones y/o tensiones entre las direcciones sindicales y las organizaciones gremiales de base en el sector de la alimentación, atendiendo a la pregunta por la incidencia de las estrategias adoptadas en el marco de las organizaciones gremiales de base sobre las tradiciones sindicales.

En esta oportunidad, planteamos un análisis centrado en las condiciones objetivas ligadas a las transformaciones registradas en la industria de la alimentación a partir de la década del 70, a fin de situar los escenarios en los que se desarrollaron las respuestas sindicales. La necesidad de considerar el análisis de la estructura económica para comprender el proceso de organización sindical se asienta sobre el supuesto que sostiene que los individuos están constituidos a partir de un conjunto de relaciones sociales, y si bien una parte de ellas son ideológicas, otra está directamente ligada a la estructura económica de la sociedad, constituyendo 
así una disposición de fuerzas objetivas (Iñigo Carrera, 2000). Para avanzar en esta dirección, proponemos un abordaje multiescalar, basado en la articulación de distintos niveles de análisis: el sector de actividad, la negociación colectiva y la empresa.

Si bien existeuna vasta bibliografía centrada enla evolución históricade la industria manufacturera en Argentina, no encontramos estudios que se enfocaran específicamente en el sector alimenticio, a excepción de algunos trabajos que han abordado su situación actual (Kandel, 2003; Trajtemberg, Varela, Medwid\& Senén González, 2007; Campos, 2012). Por ello, además de las estadísticas y la bibliografía especializada, fue necesario incorporar fuentes complementarias, como convenios colectivos y acuerdos, entrevistas en profundidad a delegados, documentos sindicales y prensa gráfica.

El artículo se estructura en tres apartados principales. En primer lugar, se abordan los cambios y continuidades que tuvieron lugar en la industria de la alimentación, comparando los años noventa y la posconvertibilidad, considerando la dinámica del mercado de trabajo, organización del trabajo y composición del capital. En el segundo apartado, se avanza sobre las características que asumió la flexibilidad laboral en el sector alimenticio a partir del análisis de la letra del CCT que rige actualmente en la actividad, convenios anteriores y acuerdos que modificaron parcialmente el convenio vigente. Por último, luego de plantear un análisis en términos de la actividad, se presenta la dinámica que asumen las relaciones laborales en un caso de estudio. Se trata de la planta que posee la empresa Mondelez en la localidad de General Pacheco en la zona norte del Gran Buenos Aires, donde se ubica una de las concentraciones fabriles más importantes del sector de la alimentación en el país. Así, a partir del análisis de fuentes primarias y secundarias, se describe la trayectoria de la empresa en Argentina, la organización del trabajo en la planta, y el modo en que impactaron los cambios del proceso de trabajosobre la fuerza laboral y la organización sindical.

\section{LA REESTRUCTURACIÓN CAPITALISTA EN TÉRMINOS SECTORIALES}

A partir de los años setenta, en el marco de las transformaciones operadas en el mercado mundial tras la crisis económica, se produjeron cambios en la industria local y particularmente en el sector alimenticio. En este contexto, la política económica implementada por la dictadura militar, basada en la liberalización de los mercados, la apertura económica al exterior y la valorización financiera impactaron sobre la actividad y, aunque no todas las ramas se comportaron del mismo modo,sufrió una importante caída.

Uno de los indicadores utilizados por algunos autores para dar cuenta de dicha retracción es el volumen físico de la producción manufacturera, que cayó más de un 10\% durante el período 1974-1983. Considerando específicamente el sector de la alimentación, sostuvieron que mientras la tasa de crecimiento anual se elevaba casi hasta un 9\% en 1974, en 1983 sólo alcanzaba un 3,8\%. Si bien el ritmo de la tasa de crecimiento anual fue oscilante durante el período, alcanzó los niveles porcentuales más bajos con la crisis económica de 1980, situación que se repitió en las restantes ramas del sector. También destacaron los cambios operados en el mercado de trabajo con la expulsión sistemática de trabajadores, que entre 1974 y 1983 ascendió a más del 34\%, y los cambios en las condiciones de trabajo evidenciados para el mismo período, por ejemplo, en el incremento de casi un 6\% de la jornada media de trabajo (Azpiazu, Basualdo\&Khavisse, 2004).

Sin embargo, no se trató de un proceso homogéneo dentro del sector manufacturero. La rama de la alimentación, junto con otros rubros como el de la refinación de petróleo y la elaboración de productos químicos, incrementó el porcentaje de personal ocupado y su participación en el valor de la producción fabril durante el período 1973-2003, mientras que otras ramas, como la industria de bienes de capital, sufrieron un marcado deterioro (Azpiazu \& Manzanelli, 2011).

Hacia la década de 1990 se profundizaron estas tendencias, y en el sector alimenticio se asistió a dos procesos: la tecnificación del sector y la compra de las empresas líderes locales por parte de empresas multinacionales (Campos, 2012). 
En relación con el primer punto, uno de los rasgos que caracterizó las políticas de racionalización empresaria en el sector fue la concentración de los cambios en la base técnica de la producción, que tendieron a economizar los requerimientos de mano de obra con el objetivo de aumentar la velocidad de los procesos, elevar los niveles de automatización y fortalecer su integración (Trajtemberg, Varela, Medwid\& Senén González, 2007). En términos organizativos, se destacaron dos transformaciones principales. Por un lado, las empresas redimensionaron sus estructuras desprendiéndose de ciertas actividades, y aunque en los años noventa las prácticas de subcontratación se circunscribieron especialmente a los servicios y/o a algunas tareas de mantenimiento o matricería, implicaron la tercerización de sectores importantes por sus dimensiones y complejidad organizativa. Por otro lado, impulsaron iniciativas de ampliación de las actividades mediante la utilización de los tiempos de espera, la rotación, la incorporación de tareas de limpieza y trabajos de otros puestos, transformando los tiempos de descanso en tiempos productivos. Asimismo, si bien las empresas mantuvieron en general estable el volumen de trabajadores ocupados, tendieron a aumentar la proporción de trabajadores precarios -eventuales y/o contratados por tiempo determinado- siguiendo una política de reemplazo. La reducción de personal afectó principalmente a las líneas de armado manual, en las que el trabajo era poco calificado y de extrema monotonía, que era el sector que concentraba -y aun lo hace- un mayor porcentaje de mujeres (Rojas, Catalano, Hernández, Rosendo \& Sladonga,1995). Sumado al aumento de la productividad de la fuerza de trabajo, se destacó una disminución del salario medio; de modo que se verificó una significativa transferencia de ingresos desde los asalariados hacia los capitalistas y por ende una creciente apropiación del excedente por parte de estos últimos.

En relación con el segundo punto, se destacó el incremento de la participación de capitales extranjeros en la economía nacional, siendo la industria de la alimentación uno de los sectores donde se concentró la inversión extranjera directa. Históricamente ésta se había desarrollado mediante un liderazgo compartido y no competitivo entre las grandes firmas locales y las empresas extranjeras. Mientras las primeras se especializaban en la fabricación de productos de marca, las segundas se destacaban en la producción de commodities (aceites y harinas) y carnes procesadas. La novedad radicó en el ingreso de firmas transnacionales que adquirieron empresas nacionales de fuerte inserción en el mercado local, con marcas reconocidas (Trajtemberg, Varela, Medwid \& Senén González, 2007). Pueden citarse diversos casos significativos, como por ejemplo Terrabusi (Manón, Lincoln, Express) que pasó en 1994 a manos de Nabisco, que luego adquirió Canale, Mayco, Capri, Royal y Tasti. En ese mismo año Bagley (Criollitas) fue adquirida por Danone. En 1995 Cadbury compró Stani, y en distintos años el ExxelGroup fue adquiriendo la panificadora Fargo, Confiterías Havanna y las heladerías Freddo.

En los años noventa la industria alimenticia adquirió una marcada centralidad, consolidándose como una de las actividades más importantes en relación con su aporte a la producción total manufacturera (26,4\%), pero también a los requerimientos de mano de obra por unidad de producción, ocupando al 26,8\% del personal total (Schorr, 2004).

Ahora bien, aunque con discusiones respecto a la profundidad y el alcance de los cambios, distintos autores coinciden en que el sistema industrial comenzó a sufrir modificaciones hacia el año 2002, siendo un punto de inflexión la política económica de devaluación de la moneda (Azpiazu \& Schorr, 2011; Grigera, 2012).

La industria fue uno de los sectores que más insistió por la salida devaluacionista, que modificó la configuración de los precios en general y de la fuerza de trabajo en particular, produciendo un cambio en el régimen de ganancias extraordinarias con su traspaso desde el sector financiero hacia el gran capital industrial (Noda \& Mercatante, 2005). En este sentido, si bien los niveles de ganancia de las grandes empresas disminuyeron en el marco de la crisis mundial de 2007/08, fueron más altos que en el mejor año de la década del noventa (1998) (Azpiazu \& Manzanelli, 2011).

Los sectores que más crecieron fueron los que se basaban en el aprovechamiento de ventajas comparativas estáticas, es decir, aquellos centrados en: la producción de alimentos y bebidas, refinación de petróleo, 
elaboración de productos químicos, armado automotriz y siderurgia. Los mismos que habían crecido en la década anterior.

Considerando el sector de alimentos y bebidas, los niveles de producción se incrementaron de forma sostenida desde fines del año 2002 hasta julio de 2007. En el bienio 2008-2009 se evidenció una leve desaceleración en el ritmo de crecimiento, influenciada principalmente por la crisis internacional, aunque en 2010 el crecimiento volvió a ser sostenido. La producción creció en volumen un 50\% entre los años 2001 y 2011 (COPAL, 2012), y hacia el año 2013 representaba el 25\% del valor agregado de la industria manufacturera, el 33,6\% de las exportaciones totales, y tenía una fuerte incidencia en el marcado de trabajo con 500.000 puestos directos (COPAL, 2013).

Otros indicadores de continuidad fueron la tendencia a la concentración económica (incidencia de las grandes firmas de una actividad en la producción total de la misma) y la centralización del capital (concentración de la propiedad de los medios de producción en pocas manos), con eje en una fuerte extranjerización de la estructura económica local (Azpiazu, Manzanelli \& Schorr, 2011). Tal como analizamosen el tercer apartado, Mondelez es un ejemplo de estos procesos, así como también podemos mencionar el caso de Arcor, que avanzó fuertemente en las compras y fusiones de empresas.

Un punto donde observamos mayores cambios fue en la creación de puestos de trabajo. La tasa de incremento de la ocupación fue particularmente alta durante los primeros años del período, más precisamente hasta el año 2007, probablemente en correspondencia con las primeras señales de la crisis económica mundial. No obstante,a diferencia del período anterior, cuyos niveles eran regresivos, la recuperación del empleo manufacturero durante el período 2003-2007 se produjo a una tasa media anual del $5,8 \%$ (Fernández \& Gonzáles, 2012). ${ }^{2}$ Esta tendencia general tuvo su correlato particular en la rama de la alimentación (ver Cuadro 1). 
CUADRO 1

Evolución del empleo en la industria de la alimentación entre 1996 y 2013

\begin{tabular}{|c|c|c|c|}
\hline & Año/ III trimestre & Empleo & Variación \\
\hline \multirow{7}{*}{$1^{\circ}$ Período } & 1996 & 271.804 & \\
\hline & 1997 & 275.664 & $1,4 \%$ \\
\hline & 1998 & 276.282 & $0,2 \%$ \\
\hline & 1999 & 267.207 & $-3,3 \%$ \\
\hline & 2000 & 260.417 & $-2,5 \%$ \\
\hline & 2001 & 250.643 & $-3,8 \%$ \\
\hline & 2002 & 238.724 & $-4,8 \%$ \\
\hline \multirow{6}{*}{$2^{\circ}$ Período } & 2003 & 251.590 & $5,4 \%$ \\
\hline & 2004 & 272.906 & $8,5 \%$ \\
\hline & 2005 & 286.073 & $4,8 \%$ \\
\hline & 2006 & 299.358 & $4,6 \%$ \\
\hline & 2007 & 315.596 & $5,4 \%$ \\
\hline & 2008 & 332.348 & $5,3 \%$ \\
\hline \multirow{5}{*}{$3^{\circ}$ Período } & 2009 & 339.064 & $2,0 \%$ \\
\hline & 2010 & 343.972 & $1,4 \%$ \\
\hline & 2011 & 357.831 & $4,0 \%$ \\
\hline & 2012 & 359.613 & $0,5 \%$ \\
\hline & 2013 & 363.111 & $1,0 \%$ \\
\hline
\end{tabular}

Fuente: Elaboración propia en base a Trajtemberg, Varela, Medwid \& Senén González (2007) y a datos del Observatorio de Empleo y Dinámica Empresarial (OEDE)

Considerando los datos del tercer trimestre de cada año, puede observarse que, luego de una etapa de crecimiento (1996-1998), en el período 1999-2002 se produjo una reducción permanente del empleo. Esta situación se revirtió hacia el 2003 y desde ese entonces nunca dejó de crecer la cantidad de asalariados registrados en el sector. En este sentido, se desataca el año 2004 como el pico de mayor crecimiento, y el año 2009 como el inicio de la desaceleración del ritmo de crecimiento que venía registrando.

\section{2- LA REESTRUCTURACIÓN CAPITALISTA EN LA INDUSTRIA DE LA ALIMENTACIÓN: UNA LECTURA DESDE EL ANÁLISIS CUALITATIVO DEL CCT}

En este apartado partimos del supuesto que sostiene que los acuerdos y convenios colectivos constituyen una de las fuentes que pueden consultarse para dar cuenta del modo en que se manifiesta históricamente la correlación de fuerzas entre capital y trabajo. Esto se debe a que establecen las condiciones que determinan la tasa de explotación del capital a través de la determinación, entre otros elementos, de la jornada laboral, las condiciones y formas de trabajo, la composición del salario, las modalidades de contratación y las vacaciones (Montes Cató, 2006). Teniendo en cuenta esto, se considera que el análisis tanto del convenio colectivo vigente, como de los acuerdos por empresa y sector, también permiten evidenciar las estrategias que utilizan los empresarios para estructurar los procesos de flexibilización laboral.

Tal como se mencionó anteriormente, durante la década de 1990 se profundizaron los procesos de concentración, centralización y extranjerización de capital, que tuvieron un fuerte impacto en el sector de la alimentación, donde las principales empresas pasaron a manos extranjeras y se inició un proceso de compras 
y fusiones. ${ }^{3}$ Las compras más importantes se iniciaron hacia finales de 1994, fecha que coincide con el año en que se firmó el CCT 244/94, que rige actualmente en la actividad, en el que se introdujeron las cláusulas de flexibilización laboral. Así, la rama de la alimentación firmaba uno de los pocos convenios colectivos por actividad del período, siendo que la práctica más recurrente en materia de negociación colectiva durante los años noventa fue la firma de acuerdos y convenios por empresa (Marticorena, 2014; Campos, 2012).

No obstante, pese a que la estructura de la negociación colectiva del sector de la alimentación muestra el predominio de los convenios por actividad, pueden destacarse algunos convenios emblemáticos por empresa como los de Bimbo, McCain y Ferrero, e incluso el denominado "Convenio Mantecol”, firmado en 2001 en la ex Cadbury (hoy Mondelez, Planta Victoria).

La expresión convenio Mantecol forma parte del lenguaje nativo. Si bien es mencionado en las entrevistas y fuentes secundarias consultadas, no existe como convenio sino como un acta que firmó el sindicato con la empresa Cadbury en 2001, cuando adquirió Mantecol (Georgalos). Se trata de un acuerdo de empresa que -según figura en la letra del documento-abarcaría solo a los trabajadores que desempeñaban tareas en el sector Mantecol. Éste fijaba condiciones desventajosas respecto al acuerdo que regía desde los años noventa en la fábrica, en tanto extendía la jornada laboral de 45 a 48 horas semanales, imponía la obligatoriedad del día sábado y anulaba el pago al $200 \%$ de las horas extras los fines de semana. La política que adoptó la empresa desde entonces fue incorporar personal en el sector Mantecol bajo el contrato de trabajo que habilitaba el acta, para luego reubicarlo en los diferentes sectores de la planta sin modificar su contrato inicial. Progresivamente, el nuevo contrato se fue extendiendo en todos los sectores, hasta alcanzar actualmente al $70 \%$ de los trabajadores de la planta. Fue firmado en el marco de la crisis que se abrió en 2001 y no se vio modificado con la compra de Cadbury a manos de Mondelez en 2010. De este modo, la centralización de la negociación colectiva que caracteriza al sector de la alimentación, expresada en la predominancia de convenios y acuerdos sectoriales, se combina con la presencia de acuerdos y/o tipos de contratos de trabajo por empresa que estipulan otras condiciones de flexibilización laboral, generalmente más regresivas.

El convenio colectivo que regula las relaciones laborales en la actividad fue firmado entre la Federación de Trabajadores de Industrias de la Alimentación (FTIA), que es la entidad de segundo grado a la que están adheridas la mayor cantidad de sindicatos de primer grado del país; ${ }^{4}$ y la Federación de Industrias de productos Alimenticios y Afines (FIPPA), en 1994. Su alcance se extiende a los trabajadores dedicados a la elaboración de distintos tipos de productos como aceitunas y encurtidos, arroz, galletitas, chocolate, bombones, café, dulces, especias y golosinas.

Entre las modificaciones más importantes que introdujo el actual convenio respecto al inmediatamente anterior (CCT89/90) pueden destacarse: (a) la polivalencia y la flexibilidad funcional en la forma de organizar el trabajo, que habilita la posibilidad de asignar al trabajador funciones y tareas diferentes a las que le son propias según las necesidades del capital; ${ }^{5}$ (b) el establecimiento de la productividad como la norma que define -al mismo tiempo que justifica- la necesidad de modernizarel marco de las relaciones laborales, habilitando la posibilidad de articular el convenio con unidades menores de negociación; (c) la flexibilización de la jornada de trabajo,incluyendola posibilidad de establecer modificacionesen los convenios y acuerdos por rama o empresa conforme las disposiciones de la ley Nacional de Empleo (ley 24.013) (jornadas promedio), y el fraccionamiento de las vacaciones en función de las necesidades de la producción; (d) la promoción de modalidades contractuales por tiempo determinado también regulada por la ley Nacional de Empleo; y (e) la incorporación de incentivos y premios como mecanismos variables en la conformación y determinación del salario (aumentos dados a cuenta y/o premios, e incentivos por productividad).

Asimismo, se destaca la intención de las partes de demostrar las supuestas ventajas que representa para los trabajadores la profundización del proceso de reestructuración productiva, y de estructurar las relaciones de trabajo a partir de procesos destinados a restringir las acciones de conjunto de los trabajadores, ocultando las contradicciones entre capital y trabajo. Esta situación se evidencia con más claridad enel artículo denominado "fines compartidos": 
“Ambas partes coinciden en la necesidad de modernizar el marco de las relaciones laborales, con el objeto de adecuarlo a las condiciones de competitividad de la economía y al mejoramiento real del trabajador. Para ello, son plenamente conscientes de que el mejoramiento de las condiciones de trabajo y de vida de los trabajadores, así como la promoción del empleo, sólo podrán ser el producto de un mayor desarrollo de la industria de la alimentación, bajo un esquema de organización que tienda a la productividad" (Artículo 4, CCT, 244/94).

El antagonismo entre capital y trabajo queda oculto en el convenio detrás del intento de la patronal por presentar sus intereses propios como generales, escondiendo la búsqueda de incrementar sus ganancias mediante una mayor explotación de la fuerza de trabajo.

También pueden mencionarse otros artículos del convenio en los que se refuerza la necesidad de mantener alejadas del lugar de trabajo las situaciones conflictivas y de eventual indisciplina que pudieran perturbar el normal desarrollo de las actividades productivas. De ahí la incorporación de la figura de "paz social” (Artículo 4), que también aparece con cierta recurrencia en los acuerdos de sector celebrados con posterioridad a la firma del convenio, como otra de las herramientas tendientes a ocultar el antagonismo estructural entre trabajo y capital, y avanzar hacia la formación de trabajadores dóciles y con sentido de pertenencia a la empresa.

Además, a fin de evitar las posibilidades de que se desarrollen conflictos abiertos, aparecen en el convenio diversos mecanismos burocráticos para dilatar el tiempo de su resolución. Entre ellos se destaca la Comisión Paritaria Permanente (CPP), que es un órgano de negociación mixta entre el sindicato y las empresas para dirimir las diferencias. Si bien la sanción de esta comisión se concretó en el convenio firmado en 1989, en el que rige actualmente se introdujeron algunos cambios. Se especificaron las funciones que debía cumplir el organismo, que incluía la intervención no sólo en las diferencias que pudieran surgir a partir de las interpretaciones del convenio colectivo sino también a partir de cualquier otra causa, bajo el título "Normas de procedimiento en conflictos". También, se aclaraba que la intervención de la comisión en cuestiones de carácter individual quedaba sujeta a la decisión de las partes y a que los trabajadores siguieran el procedimiento de queja establecido en el convenio. Asimismo, se mencionaba que no podían adoptarse medidas de acción directa en los conflictos sometidos a la CPP. Así planteada, la cláusula estaba orientada a evitar las acciones conjuntas de los obreros, individualizar los reclamos y, en definitiva, generar una correlación de fuerzas más favorable para el sector empresarial.

De igual modo, esto puede observarse a partir de la centralidad que se le asigna en el convenio a la figura de los supervisores en la gestión de los reclamos de los trabajadores:

"Los delegados de sección atenderán exclusivamente los problemas de la sección que representan, buscando la solución con el supervisor respectivo. Cuando el problema no fuera resuelto satisfactoriamente, el delegado solicitará el permiso del supervisor y lo pondrá en conocimiento del miembro de la CRI (Comisión de Relaciones Internas) más próximo. (...) La actuación del delegado de sección a que se refiere el presente artículo, queda supeditada a que previamente las reclamaciones de tipos individuales o peticiones particulares (...) sean efectuadas directamente por el interesado ante el supervisor inmediato" (Artículo 66, CCT 244/94).

A partir de la cita puede observarse la tendencia a individualizar los reclamos entre los trabajadores y los supervisores, resaltando la figura de estos últimos sobre los delegados de sector. A su vez, es de destacar la infinidad de instancias previas que dispone en convenio antes de que un reclamo particular sea tratado por la comisión interna. ${ }^{6}$

Incluido como uno de los logros centrales de la actual dirección del sindicato (revista Nuestra VOZ, 2014), el convenio flexibilizador de los años noventa (CCT 244/94) se mantuvo sin modificaciones hasta el año 2003, cuando se sancionó un acuerdo entre la FTIA y la FIPPA (No 66.142/97) que lo modificaba parcialmente. Su relevancia radica en que no se limitó a modificar las retribuciones mínimas, sino que también introdujo cambios sobre las condiciones de trabajo y contratación. 
Por un lado, mantuvo los mecanismos variables en la conformación del salario establecidos en el convenio previo. Pero si el CCT 244/94 contemplaba premios por productividad, el presente acuerdo incorporaba, además, otros incentivos compensatorios sobre el salario mínimo. En la letra del acuerdo se indicaba:

"Podrán imputarse los aumentos dados a cuenta de futuros aumentos, tickets canasta, premios, beneficios no remunerativos, presentismo, productividad $\mathrm{v}$ adicionales, hasta lograr la conformación de las retribuciones mínimas de cada una de las categorías determinadas" (Anexo I, Acuerdo No 66.142/97).

El acuerdo también introdujo modificaciones en lo que respecta al fraccionamiento de las vacaciones, otorgando a la patronal una mayor libertad para la organización del tiempo de trabajo y descanso de los trabajadores. Por otra parte, y conforme a lo dispuesto por la ley de reforma laboral 25.013, sancionada por el menemismo, que habilitaba la ampliación del período de prueba hasta 6 meses por convenio colectivo, el acuerdo modificó la duración del período de prueba, extendiéndolo de 3 a 6 meses en las empresas grandes y de 6 a 12 en el caso de tratarse de empresas pequeñas (Anexo IV).

Asimismo, estableció un descuento obligatorio a favor de cada entidad gremial de primer grado adherida a la FTIA, denominado "aporte solidario". Se trata de una suma equivalente al $2 \%$ sobre las remuneraciones mensuales de los trabajadores comprendidos por el CCT 244/94, estuvieran o no afiliados al sindicato, y siendo la parte empresaria el agente de retención de dicho aporte. A diferencia de la tendencia predominante, donde el aporte obligatorio de los no afiliados resulta un porcentaje mayor que el de los afiliados (Marticorena, 2014), en el sindicato de la alimentación es menor. Mientras el descuento compulsivo es del 2\%, la cuota sindical para los afiliados duplica este valor, representando el 4,5\% del salario. Siguiendo a Marshall \& Perelman (2004), quienes sostienen que cuanto menor es la diferencia entre el descuento compulsivo y la cuota sindical mayor sería el incentivo a afiliarse, ya que por un costo diferencial mínimo se accede a los beneficios exclusivos asociados con la condición de afiliado, en este caso la relación opera de un modo contrario. Así, la diferencia entre el aporte regular compulsivo y la cuota sindical puede plantearse como un mecanismo de desincentivo a la afiliación. Más aun, el acuerdo de 2003 establecía que los sindicatos destinatarios del aporte solidario podrían disponer del pago del mismo a quienes ya venían realizando sus aportes correspondientes, aunque, en un acuerdo posterior del año 2013 se definió que los trabajadores afiliados a los sindicatos de primer grado compensarían el aporte solidario, hasta su concurrencia, con la cuota sindical.

Así planteado, el descuento regular compulsivo permite a la dirección del sindicato resolver en términos financieros el problema de la desafiliación, ${ }^{7}$ al mismo tiempo que regula le necesidad de diseñar políticas de incentivo para la incorporación de nuevos afiliados. Hablar de desincentivación a la afiliación no resulta extraño si se considera que al mismo tiempo que tienen obligaciones (aportan el 4,5\% de su sueldo al sindicato), los afiliados cuentan con derechos, entre los que se destaca la habilitación para votar en las elecciones generales. Recién hacia 2014, en el marco de un giro más profundo en la estrategia de la dirección del sindicato, observamos un cambio en relación a este punto, expresada en un mayor incentivo a la afiliación mediante la publicación de campañas abiertas de reclutamiento de miembros. Como una lectura posible de este cambio, planteamos el peso creciente que venían cobrando los sectores opositores a la dirección del sindicato en las grandes fábricas y la necesidad de la dirección de relegitimarse ante las bases. ${ }^{8}$

Finalmente, el nuevo acuerdo también fijó una contribución empresaria a favor de la FTIA, calculada mensualmente según el número de trabajadores comprendidos por el convenio colectivo, destinada atareas de formación profesional y capacitación de los trabajadores.

En el mismo acuerdo en que la entidad sindical aprobaba medidas que habilitaban un mayor avance del capital sobre el trabajo,sancionaba la generalización de aportes y contribuciones a su favor tanto por parte de los trabajadores comprendidos en el convenio colectivo, como por parte del sector empresario, que de esta forma compensaba la mayor disposición adquirida sobre la fuerza de trabajo a través del nuevo acuerdo. 


\section{LA MATERIALIDAD DEL PROCESO DE TRABAJO EN UN CASO DE ESTUDIO}

\section{La trayectoria de la empresa en el país}

Si bien en Argentina es más conocida por su denominación anterior, Kraft Foods, en octubre de 2012 la firma decidió dividir su negocio de alimentos y dio lugar al surgimiento de Mondelez International, creando dos compañías, una de snacks con alcance global, y otra específicamente para el sector de alimentos del mercado norteamericano, que conservó su denominación anterior.

Actualmente, ocupa el primer lugar en la fabricación global de chocolates y galletitas, y es la segunda empresa productora de chicles, liderando a su vez los mercados de caramelos duros, bebidas en polvo y premezclas. Con más de 230 años de antigüedad, cuenta con 80 plantas ubicadas en 165 países del mundo, más de 110.000 empleados, y factura anualmente 35 millones de dólares.

Mondelez Argentina es una firma subsidiaria de Mondelez Internacional, cuyas responsabilidades comerciales se extienden a distintos países del Cono Sur, como Chile, Uruguay y Paraguay. ${ }^{9}$

Actualmente, cuenta con cuatro establecimientos industriales: (a) Planta Pacheco (ex Kraft-Terrabusi), ubicada en el partido de Tigre, provincia de Buenos Aires, que se dedica a la producción de galletitas y chocolates; (b) Planta Victoria (ex Cadbury-Stani), ubicada en el partido de San Fernando, provincia de Buenos Aires, que se dedica a la elaboración de chocolates, chicles y caramelos; (c) Planta Villa Mercedes (ex Alimentos Especiales S. A.), ubicada en la ciudad de Villa Mercedes, provincia de San Luis, que se dedica a la producción de bebidas en polvo y postres para preparar; y (d) Planta Las Heras (ex Molinos Río de la Plata), ubicada en el partido de General Las Heras, provincia de Buenos Aires, que se dedica a la elaboración de caramelos. En 2013, luego del extenso conflicto que tuvo lugar en la planta de la ex Kraft-Terrabusi en 2009, que involucro bloqueos en las oficinas administrativas que allí funcionaban ${ }^{10}$, la firma decidió su traslado a la localidad de Villa Adelina del partido de San Isidro.

La historia de Mondelez en el país involucró más de 9 fusiones y adquisiciones que tuvieron inicio en la década de 1980. En el marco de la profundización de la extranjerización de la industria local y de las tendencias a la adquisición de firmas preexistentes, la empresa Nabisco se instaló en Argentina hacia el año 1981, tras la fusión con Standars Internacional que representaba en el país a Fleischmann (Royal). Luego, en 1990 ocurrió lo mismo con Kraft Foods, dando inicio a la historia de la compañía en el país. Durante la década de 1990, tal como ocurrió en muchos otros sectores industriales y de servicios, ambas firmas comenzaron un proceso de concentración, adquiriendo una gran cantidad de empresas argentinas tradicionales. Nabisco Royal Argentina Inc. adquirió Terrabusi en 1994 y Vizzolini, Canale, Mayco y Capri en 1996. Mientras que Kraft Foods hizo lo mismo con Suchard y Alimentos Especiales, la firma elaboradora de bebidas en polvo, y terminó relanzando en el país las marcas Tang y Clight. Hacia el año 2000, Philip Morris absorbió Nabisco a través de Kraft Foods, su división alimenticia separada de los negocios tabacaleros, y finalmente en 2010 Kraft Foods adquirió también Cadbury. ${ }^{11}$

Luego de instalarse en el país, tanto Nabisco como Kraft Food siniciaron un proceso de reorganización de la producción. Tras adquirir marcas como Canale, Mayco, Capri, Royal, Terrabusi y Tasti, Nabisco cerró sus plantas e inició un proceso de concentración de las actividades productivas.

El cierre del histórico establecimiento de Terrabusi ubicado en la calle San José 1060, en el barrio de Constitución, Ciudad Autónoma de Buenos Aires, se produjo en 1998. La relocalización de las actividades productivas que allí se desarrollaban a la planta de General Pacheco fue comunicado en febrero de 1998 y, según el acuerdo firmado entre la empresa y el sindicato, el traslado se llevó adelante en distintas etapas a lo largo de ese mismo año (Expte. $\mathrm{N}^{\circ}$ 66527/98).

Inmediatamente después, en mayo de 1999, se comunicó el traslado de los trabajadores de la ex Fleischmann - Royal (Planta Munro), ubicada en Avenida Bernardo Ader 2754, Munro, Provincia de Buenos Aires. A diferencia del caso anterior, este traslado dejó como saldo la pérdida de puestos de trabajo, dado que 
el acuerdo no contemplaba el traslado de todos los trabajadores. Según consta en el artículo noveno de dicho acuerdo, "los trabajadores no trasladados serán indemnizados conforme la 'indemnización por antigüedad prevista en el Art. 245 de la L.C.T” (Expte. No 041-068.069/99).

En 2003, ya bajo la dirección de Kraft Foods, la empresa comunicó al sindicato su decisión de reorganizar las actividades industriales, discontinuando las actividades de las plantas de la ex Suchard, ubicada en Avenida Rabanal 3220, Villa Soldati, Ciudad de Buenos Aires, y cerrando definitivamente la Planta Munro. Así como en el caso anterior, nuevamente se registraron despidos y la desvinculación de delegados congresales. En este último caso el argumento fue más directo, la empresa reconoció que la discontinuidad de las actividades productivas respondía a la necesidad de obtener una permanente optimización de los recursos para hacer frente a la competencia del mercado (Acuerdo Expte. No 1.072.360/03). La contracara de la optimización de los recursos empresariales fue la reducción del plantel de trabajadores, el aumento de la concentración de trabajadores en un mismo espacio laboral (todos los cierres de plantas registrados implicaron la relocalización de las actividades en la planta de General Pacheco), la desvinculación de delegados sindicales y el cierre de líneas enteras.

Según Ciccolella \&Vecslir (2012), el proceso de metropolización industrial, es decir, de relocalización de la actividad industrial desde los espacios centrales hacia los partidos de la segunda y tercera corona del AMBA (en este caso hacia el partido de Tigre), se profundizó durante los años noventa, respondiendo a distintos factores: la disponibilidad de suelo comparativamente más barato, beneficios impositivos, menores controles urbanísticos y mejoras en la accesibilidad.

Las compras y fusiones no se detuvieron durante la posconvertibilidad. A principios de 2010 Kraft Foods adquirió la empresa inglesa Cadbury, con la intención de liderar el sector en América Latina y otros países en los que estaba instalada (Página 12, 19/01/2010). A partir de esta compra la firma pasó a controlar un cuarto del mercado argentino y se convirtió en un competidor de peso para Arcor, que lideraba el sector de golosinas en el país (La Nación, 20/01/2010), ingresando en las categorías de chicles y caramelos, en las que Cadbury controlaba el mercado con marcas como Beldent y Halls, y reforzando la categoría de chocolates.

Luego, en octubre de 2014, Molinos Río de la Plata y Mondelez decidieron hacer un canje de sus marcas de fideos y golosinas en una operación de más de 220 millones de dólares. Molinos compró los activos de Mondelez vinculados al negocio de las pastas, con marcas como Don Felipe, Vizzolini y Canale, y se quedó con la planta que Mondelez poseía en Tres Arroyos. Mondelez, por otra parte, se quedó con su negocio de los caramelos, con marcas como Billiken y las pastillas DRF, y con la planta de la localidad bonaerense de General Las Heras (Clarín, 04/10/2014).

Para cerrar este apartado que, en la búsqueda por caracterizar la trayectoria de Mondelez en el país, abordó los procesos de centralización de capital que definieron su actividad comercial y productiva en las últimas dos décadas, es importante considerar, además del impacto que tuvieron las compras y fusiones sobre la organización del trabajo, el aspecto simbólico que involucran las relocalizaciones y sucesivos cambios de nombre. La acumulación de experiencias de explotación, de lucha y de organización que fue configurando el colectivo de trabajadores de cada unidad productiva se ve interrumpido tras el cierre abrupto de una planta. Lo mismo ocurre con los cambios de nombre que se producen tras las fusiones y compras de empresas. En este punto surgía la duda acerca de cómo nominar al caso que aquí estudiamos. Aunque optamos por remitirnos a su denominación actual (antecedido por el nombre anterior para distinguirla del resto de las plantas que posee la firma en Argentina), no desconocemos que detrás de dicha marca quedan ocultas e invisibilizadas las luchas protagonizadas por los obreros de Terrabusi durante los años setenta y ochenta, y de Kraft Foods durante los años dos mil. En ese sentido, borrar el nombre es como borrar parte de la tradición construida por los obreros a lo largo de los años.

La reestructuracióndel trabajo enKraft-Mondelez

El establecimiento de Kraft-Mondelez, conocido como Establecimiento Modelo Terrabusi, fue construido en 1963 y es una de las más grandes y complejas plantas elaboradoras de galletitas en el mundo. Está 
ubicado sobre la avenida Henry Ford 1134, a 400 metros de la Ruta Panamericana. En sus inmediaciones se encuentran emplazadas las plantas automotrices más importantes del país, Volkswagen y Ford, el Frigorífico Rioplatense,y el Parque Industrial Garín -en el que funcionan empresas como Alba, La Salteña, y la imprenta Madygraf (ex Donnelly), actualmente recuperada por sus trabajadores. Históricamente, esta zona ha sido el epicentro de una intensa actividad de comisiones internas y cuerpos de delegados combativos, alcanzando mayor visibilidad en la década de 1970 con la experiencia de la Coordinadora Interfabril de zona norte.

De los 4.800 trabajadores que Mondelez emplea actualmente en el país, más de 2700 trabajan en la planta de General Pacheco, de los cuales aproximadamente el 60\% son mujeres. A diferencia de otras fábricas alimenticias, incluso del resto de las plantas que posee la firma en Argentina, en la planta de Kraft-Mondelez los turnos de trabajo son rotativos. El sistema de relevos está organizado en tres turnos fijos de ocho horas cada uno, asegurando el uso intensivo de los medios de producción durante las veinticuatro horas del día. Esto responde a la necesidad de valorización del capital, dado que, tal como señala Marx:

\footnotetext{
"El capital constante, los medios de producción, si se los considera desde el punto de vista del proceso de valorización, sólo existen para absorber trabajo, y con cada gota de trabajo una cantidad proporcional de plus trabajo. En la medida en que no lo hacen su mera existencia constituye una pérdida para el capitalista, pues durante el tiempo en que permanecen inactivos representan un adelanto inútil de capital" (Marx, 2008: 308).
}

Mantener la organización del trabajo en turnos fijos puede considerarse como un triunfo de los trabajadores de Kraft-Mondelez, dado que entre las modificaciones más destacadas introducidas durante los años noventa se destacan los cambios en los tiempos de trabajo, mediante el establecimiento de las jornadas promedio, los turnos rotativos, el turno americano y el trabajo continuo, entre otros mecanismos, ampliando la disponibilidad del capital sobre el trabajo. Además de reducir los niveles de imprevisibilidad que generan los turnos rotativos y/o americanos en la organización de la vida de los trabajadores, el trabajo en turnos fijos amplia las posibilidades de generar lazos de solidaridad estables en el espacio laboral y combatir la fragmentación del colectivo de trabajo.

Las tareas se realizan con un descanso de media hora, previsto por el convenio colectivo de trabajo para quienes trabajan en turnos de horario corrido, a los efectos de comer e ir al baño. Estas interrupciones son programadas previamente por la empresa, dado que el convenio no establece mayores precisiones al respecto.

Como parte del reclamo de los trabajadores y la comisión interna de Kraft-Mondelez contra las enfermedades laborales derivadas de los movimientos repetitivos en la línea de producción, hacia el año 2012 logró implementarse un sistema de micropausas. El mismo consistió en la posibilidad de que cada trabajador cuente con una pausa por turno rotativo. Se trata de un minuto y medio en el que pueden dejar su puesto para realizar ejercicios de estiramiento, para luego regresar a la línea de producción. Considerando que se trata de una pausa por turnos rotativos, cada trabajador finalmente termina contando con un minuto y medio de descanso cada dos horas de trabajo. Aunque evidentemente no logran resolver la problemática de las enfermedades laborales, estas medidas representan pequeñas conquistas en la lucha contra la intensificación de los tiempos de trabajo.

Ahora bien, lo cierto es que la jornada laboral no presenta límites homogéneos al interior de la fábrica, y esto es particularmente evidente en el caso de los trabajadores contratados. Tal como sostiene Marx (2008), pese a que la variación de la jornada laboral oscila dentro de límites físicos y sociales, ambas dimensiones son elásticas y permiten la libertad de movimientos, de modo que es posible encontrar jornadas de trabajo de prolongaciones muy variadas. Esto observamos al comparar la situación de los trabajadores efectivos y contratados:

\footnotetext{
"Cuando entré me mataba, iba de seis a seis, justamente porque hacíamos muchas horas extras, después cuando quedé efectiva, no. Al principio sentís la presión, sentís que tenés que cumplir, te decían hoy hay extra y casi ni te preguntaban, y después la necesidad de la guita, no" (delegada de la comisión interna, diciembre de 2011).
} 
Además de operar sobre la reducción de los costos laborales, la precarización contractual habilita una mayor disponibilidad del capital sobre el tiempo de trabajo, en la medida en que, tal como se desprende del fragmento de la entrevista, la condición de contratado vuelve al trabajador más vulnerable a los requerimientos de la patronal. Así, llegaban a realizar jornadas de más de 12 horas de trabajo continuo, bajo la presión de alcanzar en un futuro próximo la tan ansiada efectivización. Por último, un tercer efecto que nos interesa destacar de la precarización contractual es la fragmentación del colectivo obrero, con las dificultades que ello implica en términos de las posibilidades de avanzar en la organización sindical en el lugar de trabajo. Desde el punto de vista de la racionalidad empresaria, el objetivo de estas medidas también consiste en dividir e incluso impulsar la competencia entre los trabajadores, a fin de individualizar el vínculo de los obreros con la empresa y disolver las solidaridades internas de la clase trabajadora.

Tanto la terciarización de actividades, como las contrataciones mediante agencias eventuales comenzaron a hacerse más extensivas en la planta a partir de la crisis de 2001. Mantener una proporción fija de trabajadores contratados por medio de agencias eventuales durante períodos prolongados de tiempo (uno o dos años consecutivos) y luego despedirlos para efectuar inmediatamente la misma cantidad de contrataciones era una práctica recurrente para la empresa. Luego del conflicto que tuvo lugar en 2009 la empresa cambio su política en relación a este punto. Reemplazó las contrataciones a través de agencias eventuales por contrataciones directas no renovables que, al cabo de seis meses, devenían en efectivizaciones.

En relación a la organización del trabajo, pueden destacarse tres grandes secciones de producción: el sector de preparación (en el que se distribuyen los insumos para los distintos productos según la composición adecuada), el de elaboración y el de envasado. La cantidad de trabajadores que ocupa cada una es variable, dado que está asociada con los ciclos estacionales de los diferentes productos.

A diferencia del sector de elaboración, en el que la preparación de la masa y el horneado tiene pausas, en la sección de envasado se imponen ritmos de trabajo continuos. En cada línea de producción se exige determinada cantidad de golpes por minuto que equivalen a paquetes de los distintos productos. Durante la década de 1990 se cambiaron algunas máquinas que implicaron el incremento del número de golpes por minuto y por ende de los ritmos de trabajo. Por ejemplo, en aquel entonces, en la Línea Express se registraban 45 golpes por minuto, en la Línea Club Social 200 golpes, y en la Línea Chocolate (Rodhesia, Tita, etc.) 120 golpes (Kandel, 2003). Asimismo, en contraste con el sector de amasadora (elaboración), en el que se requiere la manipulación de elementos pesados y se emplea fundamentalmente fuerza de trabajo masculina, las mujeres están concentradas en el sector de envasamiento, donde el trabajo es manual, poco calificado y de extrema monotonía.

Para caracterizar la organización del trabajo en la planta también es importante mencionar las jerarquías que operan en la materialización del control sobre los trabajadores. En este orden, cada línea de producción se encuentra bajo el control de un líder de sector, que son los que ejercen el mando en nombre del capital durante el proceso de trabajo. Concretamente, son los encargados de controlar los ritmos de trabajo, los horarios, los tiempos de descanso y la asignación de tareas, es decir, son quienes ejercen cotidianamente el control directo sobre los trabajadores. Además de los líderes de sector, el personal jerárquico a nivel de planta también involucra a los coordinadores, los managers y los gerentes de Recursos Humanos.

Por último, en relación a la composición de la fuerza de trabajo es importante destacar que las mujeres son mayoría en la planta de Kraft-Monedelz. Para el año 2012 representaban el 60\% de la fuerza de trabajo de un total de 2700 trabajadores. El carácter mayoritario del empleo femenino es un rasgo que caracterizó históricamente no sólo a la fábrica en cuestión, sino también a la rama de alimentación en general. Haciendo referencia a esta particularidad del sector, un representante del sindicato nos decía:

"históricamente [hemos tenido mucha cantidad de mujeres en el gremio] porque la mujer trabajaba en lo que eran las líneas, donde se necesitaba mucho trabajo manual y el hombre es muy torpe, entonces eran todas mujeres. En muchos casos era muy artesanal. Por ejemplo, las rosetas de los huevos de pascua las hacían las mujeres, era todo artesanal porque lo hacían todo con la manga. Evidentemente todo fue cambiando, pero igual sigue habiendo muchas mujeres porque nosotros seguimos 
teniendo en las líneas mucho trabajo manual, clasificación de las galletas o de lo que sea, tenemos muchas mujeres. Ha bajado, porque nosotros teníamos antiguamente creo que era un 75 a 25, hoy estará 50 y 50" (Miembro del Consejo Directivo del Sindicato de Trabajadores de Industrias Alimenticias -STIA-, mayo de 2014).

Además del peso que históricamente tuvieron las mujeres en términos de la composición del trabajo en el sector, de la afirmación de la cita se desprenden otros elementos interesantes. Por un lado, la naturalización de que las mujeres estén concentradas en sectores y puestos de trabajo manual y poco calificado, en base a la atribución de ciertas cualidades físicas y sociales particulares. Por otro lado, que los cambios tecnológicos y productivos no modificaron esta asociación lineal entre trabajo manual y empleo femenino, que se mantiene como un rasgo de continuidad indiscutido. Y finalmente, la reducción registrada en la brecha entre el empleo femenino y el empleo masculino a favor de este último que tuvo lugar en los últimos años, habilita la pregunta acerca de si existe alguna asociación entre el crecimiento del empleo masculino, la tendencia creciente a la tecnificación y las dificultades que encuentran las mujeres para acceder a categorías más altas, que se plantea como un reclamo histórico de las trabajadoras y la Comisión Interna de Kraft-Mondelez.

A pesar de que el convenio no establece restricciones al respecto, las trabajadoras tienen menos posibilidades que los varones para acceder a las categorías más altas:

“acceder a una categoría es difícil ya por el hecho de ser mujer y eso lo vi en todas las fábricas en las que trabajé. La diferencia se nota y la empresa la hace notar. Acá en Kraft la empresa hace notar la diferencia” (delegada de la comisión interna, diciembre de 2011).

Igualmente ocurre en términos salariales ya que, aunque el convenio establece que las condiciones referentes a las retribuciones salariales deben aplicarse indistintamente a ambos sexos (Art. 26 del CCT 244/94), las mujeres cobran menos que los hombres por el mismo trabajo. Aunque, en gran parte, esta situación responde a las dificultades que encuentran las mujeres para el acceso a las categorías altas, dado que la escala salarial se organiza justamente en función de las jerarquías que establece el sistema de categorías.

A diferencia de lo que ocurre en otros sectores, en la alimentación no se han observado cambios sustanciales en términos de exclusiones o reducciones en la escala de categorías de convenio (Marticorena, 2015), aunque sí está explícitamente planteada la polivalencia y la flexibilidad funcional que habilita la movilidad de los trabajadores respecto de las tereas definidas por aquellas. De hecho, el convenio plantea que las categorías no deben interpretarse como estrictamente restringidas, en lo funcional, a las definiciones que expresan, de modo que es muy común encontrar trabajadores que han desarrollado tereas correspondientes a categorías superiores sin el debido reconocimiento de la empresa. Así lo planteaba una delegada sindical entrevistada:

\footnotetext{
“Tenemos una compañera que sabía manejar todas las máquinas y no le querían dar la categoría de oficial porque tenía tarea liviana, la definitiva, no podía volver a tocar una máquina nunca más, terminó con clavos en la espalda, no sé cuántas operaciones, producto de cómo laburaba, producto de los ritmos. La afectó en su vida personal, después de un embarazo de riesgo como el que tuvo no podía levantar a los pibes. Y después vos le estás regateando la categoría, ya pasa por odio al obrero, me entendés, ya pasa lo humano, no sé cómo explicarte" (delegada de la Comisión Interna, diciembre de 2011).
}

Además de reflejar el impacto de las condiciones y los ritmos de trabajo sobre la salud y la vida de los trabajadores, la cita permite evidenciar las dificultades que encuentran las mujeres para conseguir ascensos en las categorías, así como la discrecionalidad en su otorgamiento por parte de la empresa. Pese a que el convenio define la promoción a categorías superiores en la base a la disponibilidad de vacantes, la antigüedad y el orden de mérito ("eficacia" para el puesto), en la práctica terminan primando criterios ad-hoc, que fomentan la competencia entre los trabajadores y transforman un derecho adquirido en un mecanismo de presión de la patronal. De este modo, la promoción es un reclamo extendido y de marcada actualidad en la planta que, como vimos, se plantea fundamentalmente como una problemática de género. 


\section{Reflexiones finales}

A lo largo del artículo hemos buscado describirla situación de la industria alimenticia local entre los años noventa y la actualidad. Para ello, propusimos un abordaje multiescalar, basado en la articulación de distintos niveles de análisis (el sector de actividad, la negociación colectiva y la empresa), con el objetivo de profundizar la discusión en torno a los cambios y continuidades que podían registrarse.

En relación al sector de actividad, observamos que, en correspondencia con las necesidades de la reestructuración capitalista, la evolución del sector alimenticio se vio atravesada por dos procesos principales. Por un lado, una creciente tecnificación, que influyó sobre la organización y las condiciones de trabajo, promoviendo una mayor intensificación, flexibilización y pérdida de puestos de trabajo (subcontratación y tercerización). Por otro lado, la concentración económica y la centralización del capital, que derivó en una mayor monopolización y extranjerización del sector, mediante las compras y fusiones de empresas locales a manos de firmas extranjeras. Estos procesos no se detuvieron durante la posconvertibilidad, y sólo se identificó el crecimiento del empleo como un elemento de cambio respecto a los años noventa. Así, a diferencia de otros sectores industriales que sufrieron un fuerte deterioro con el neoliberalismo, la alimentación se consolidó como una actividad de peso y fuerte dinamismo en términos de su aporte a la producción total manufacturera y los requerimientos de mano de obra.

El análisis comparativo del convenio colectivo y acuerdos que rigen en la actividad nos permitió identificar los cambios introducidos por el modelo neoliberal en las condiciones de compra-venta de la fuerza de trabajo, destacándose la polivalencia y flexibilidad funcional en la organización del trabajo, la flexibilidad de la jornada de trabajo, la variabilidad en la composición del salario y las modalidades contractuales por tiempo determinado, como las principales. Asimismo, tal como también destacaron otras investigaciones, observamos que la negociación colectiva en el sector de la alimentación durante la posconvertibilidad no sólo no revirtió la pérdida de derechos experimentada por los trabajadores en los años noventa, sino que profundizó la flexibilización laboral, expresada en términos salariales, contractuales, y en lo que respecta a la determinación y distribución del tiempo y la organización del trabajo.

Finalmente, el análisis de estas dimensiones a partir de un caso de estudio permitió comprobar las dinámicas generales observadas, así como concretizarlas en disputas específicas entre capital y trabajo. El análisis de la trayectoria de Mondelez en el país nos permitió identificar su protagonismo en los procesos de compras y fusiones de empresas locales (con los consiguientes cierres y relocalizaciones de plantas), que se vio fuertemente impulsado durante los años noventa y continuó en los años subsiguientes. Asimismo, el estudio de algunas dimensiones que hacen al proceso de trabajo en la planta más importante que posee la empresa en el país contribuyó a distinguir conquistas parciales de los trabajadores en torno, por ejemplo, a la definición de la jornada laboral y las condiciones de contratación fijadas por el convenio colectivo, en el marco de una relación conflictiva entre capital y trabajo, que se estructura en base a reclamos históricos, tales como el sistema de categorías y la igualdad salarial entre mujeres y hombres.

Considerando lo dicho, entendemos que el análisis propuesto contribuyó a echar luz sobre el impacto de las transformaciones industriales en un sector particular de la industria sobre las condiciones de compra y venta de la fuerza de trabajo expresadas en la negociación colectiva. A su vez, permitió estudiar de qué modo dichas reglamentaciones se implementaban finalmente a nivel del lugar de trabajo, donde su aplicación se definía en función de las relaciones de fuerzas específicas que allí se desarrollaban. Así, el análisis planteado pretendió ser un aporte en orden de ampliar la caracterización del escenario en el que se desenvuelve la acción sindical y la conflictividad laboral en un sector particularmente dinámico para la economía local. 


\section{REFERENCIAS}

Azpiazu, D. \& Manzanelli, P. (2011). "Reinversión de utilidades y formación de capital en un grupo selecto de grandes firmas (1998-2009)", en Realidad Económica, Buenos Aires.

Azpiazu, D. \& Schorr, M. (2011). "La industria argentina en las últimas décadas: una mirada estructural a partir de los datos censales”, Realidad Económica, Buenos Aires.

Azpiazu, D.; Manzanelli, P. \& Schorr, M. (2011). "Concentración y extranjerización en la economía argentina en la posconvertibilidad (2002-2008)", Cuadernos del CENDES, Vol. 28, No. 76.

Azpiazu, D; Basualdo, E. \& Khavisse, M. (2004). El nuevo poder económico en la argentina de los 80, Buenos Aires, Siglo XXI.

Cambiasso, M. (2016). "Organización sindical en el lugar de trabajo e izquierdas en la Argentina reciente: la Comisión Interna de Kraft-Mondelez (ex Terrabusi)” en Trabajo y Sociedad, n² 26: Santiago del Estero.

Cambiasso, M. (2015). Tradición de organización y estrategias sindicales. Un estudio sobre la organización sindical en la fábrica alimenticia Kraft-Mondelez (ex Terrabusi) en la post-convertibilidad. Tesis Doctoral. Facultad de Ciencias Sociales, Universidad de Buenos Aires.

Campos, Julia (2011). "Volver, volver... las relaciones intrasindicales en la determinación de la negociación colectiva. El caso de la industria de la alimentación 2006-2011”, en Palomino, H.; Bleynat, I.; Garro, S.; Oyarzo, L.; \& Sánchez, R. (Eds.).Trabajadores y conflictos laborales en el nuevo régimen de empleo de la Argentina. Buenos Aires (En prensa).

Ciccolella, P. \& Vecslir, L. (2012). Dinámicas, morfologías y singularidades en la reestructuración metropolitana de Buenos Aires, en Revista Iberoamericana de Urbanismo, núm. 8, Barcelona.

Fernández, A \& González, M. (2012). "La desigualdad en los ingresos laborales. Su evolución en la posconvertibilidad”, en Apuntes para el cambio, Año 2, No 3, mayo-junio, Buenos Aires.

Grigera, Juan (2012). Reconsideración del proceso de desindustrialización en Argentina (1976-2001), Tesis doctoral, Facultad de Filosofía y Letras, Universidad de Buenos Aires.

Iñigo Carrera, N. (2000). La estrategia de la clase obrera, 1936, Buenos Aires, La Rosa Blindada-PIMSA.

Kandel, E. (2003). Las relaciones de género en una empresa de la industria de la alimentación en la Argentina en la década de 1990 (Caso T.), Tesis de Maestría, Centro de Estudios Avanzados, Universidad de Buenos Aires.

Marshall, A. \& Perelman, L. (2004), “Sindicalización: Incentivos en la normativa sociolaboral”, en Cuadernos del IDES, Buenos Aires.

Marticorena, C. (2014). Trabajo y negociación colectiva. Los trabajadores en la industria argentina, de los '90 a la posconvertibilidad. Buenos Aires: Imago Mundi.

Marx, K. (2008). El capital, Tomo I, Buenos Aires, Siglo XXI.

Montes Cató, J. (2006). Dominación y Resistencia en los espacios de trabajo. Estudio sobre las relaciones de trabajo en empresas de telecomunicaciones, Tesis Doctoral, Facultad de Ciencias Sociales, Universidad de Buenos Aires.

Noda, M. \& Mercatante, E. (2005). “El plan K: un neoliberalismo de 3 a 1”, en Revista Lucha de Clases $\mathrm{N}^{\circ} 5$, Buenos Aires.

Rojas, E.; Catalano, A. M.; Hernández; D.; Rosendo, R.; \&Sladonga, M. (1995).” Cambio tecnológico y mercado de trabajo. Los sindicatos y la tecnología: cambios técnicos y de organización en las industrias metalmecánicas y de la alimentación en Argentina", OIT-ACDI-Proyecto Regional, Vol. I y II.

Schorr, M. (2004). Industria y nación: poder económico, neoliberalismo y alternativas de reindustrialización en la argentina contemporánea, Buenos Aires Edhasa, Buenos Aires.

Trajtemberg, D.; Varela, H.; Medwid, B.\&Senén González, C. (2007). "Relaciones laborales en el sector de la alimentación”, en Trabajo, Ocupación y Empleo, Serie de Estudios N 6, Ministerio de Trabajo y Seguridad Social, Buenos Aires.

Varela, P. \& Lotito, D. (2009). "LA LUCHA DE KRAFT-TERRABUSI. Comisiones Internas, izquierda clasista y 'vacancia' de representación sindical”, en Revista Conflicto Social, No2, Buenos Aires. 
CCT 244/94

ССТ $89 / 90$

Acuerdos variosentre FTIA y FIPPA

Diarios Clarín, Página 12, La Nación: noticias varias

Informes estadísticos de la Coordinadora de Industrias de Productos Alimenticios (COPAL)

Revista sindical "Nuestra VOZ”, 2014

Ley Nacional de Empleo (24.013) y ley de reforma laboral 25.013.

Observatorio de Empleo y Dinámica Empresarial (OEDE) del Ministerio de Trabajo, Empleo y Seguridad Social (MTEySS)

Informe de coyuntura del Observatorio del Derechos Social de la CTA, 2014

Entrevista a delegada de la Comisión Interna de Kraft- Mondelez, diciembre de 2011.

Entrevista a miembro del Consejo Directivo del STIA, mayo de 2013.

\section{Notas}

1 Algunas excepciones son: (a) Arcor, que es una empresa multinacional argentina que adquirió también otras empresas de menor tamaño; y (b) Molinos, que fue comprada por la firma local Pérez Companc. Por su parte, el sector de pequeñas y medianas empresas continuó siendo en general de capitales nacionales.

2 Son 39 sindicatos de primer grado, la mayoría de los cuales cuenta con personería gremial. La federación abarca también 8 convenios colectivos y representa a más de 55.000 trabajadores. Tiene capacidad legal para firmar convenios colectivos y acuerdos, y de hecho es la organización que lo hace en la mayoría de los casos, en el marco de una actividad en la que a diferencia de otras donde predominan los acuerdos por empresa- la estructura de la negociación colectiva muestra un carácter más centralizado, con presencia mayoritaria de los convenios de actividad, expresado no sólo en términos de la cantidad de trabajadores cubiertos, sino también en la cantidad de convenios firmados y de sectores representados. El convenio más importante es el 244/94, pero también negoció en los últimos años otros 6 convenios de menor relevancia, y cuenta también con 4 convenios de empresa: Mc Cain, Bimbo, Ferrero y Alpesca S.A., además del acuerdo con emprendimientos Bragado (Trajtemberg, Varela, Medwid\& Senén González, 2007).

3 "las partes acuerdan que los empleadores tendrán la facultad de disponer los cambios en las modalidades de trabajo que consideren necesarias para el mejor desenvolvimiento de las actividades productivas y para optimizar la utilización de las variables intervinientes. Asimismo, podrán disponer cambios de tareas siempre que se respete la remuneración de la categoría a la cual pertenezca el trabajador, ya sea en función de mayor o menor jerarquía o en sectores distintos a los cuales se encuentra asignado, sin que ello implique reconocimiento de la categoría superior en que eventualmente se desempeñe" (CCT 244/94, art. 41).

4 En la fábrica, la figura del delegado de sector se ve superpuesta con la del delegado de la comisión interna.

5 A partir de las entrevistas realizadas y los documentos consultados en el marco del trabajo de campo, pudo observase cómo durante los años noventa la desafiliación no sólo respondió a la pérdida de empleo en el sector, sino que los trabajadores también recurrieron a la desafiliación como una modalidad de expresar su descontento con la gestión de su dirección, en un sindicato donde históricamente habían sido altos los niveles de afiliación. Según se desprende de las entrevistas con miembros del consejo directivo, para el año 2013, de un total estimativo de 15.000 trabajadores en condiciones de sindicalizarse, sólo 6.000 estaban efectivamente afiliados al sindicato (40\%).

6 Para un análisis más extendido sobre los cambios registrados en la política de la dirección del sindicato en los últimos años, ver Cambiasso (2016).

7 Las principales marcas y categorías en las que se destaca son: bebidas en polvo -Tang y Clight-, chicles y caramelos -Beldent, Bubbaloo, Bazooka, Halls, Lengüetazo, Palitos de la Selva, Yapa, Media Hora, Yummy, Billiken y DRF-, chocolates -Milka, Cadbury, Shot, Toblerone, Tita, Rhodesia, Habanitos y Snacky-, alfajores -Terrabusi, Milka, Tita, Oreo, Pepitos, Mantecol y Shot-, galletas dulces y rellenas -Oreo, Pepitos, Melba, Duquesa, Terrabusi Variedad, Lincoln y Manón-, crackers -Express, Club Social, Cerealitas y Mayco, y premezclas (flanes, gelatinas, polvo de hornear, mousse y postres) -Royal-, participando en el mercado local con más de 400 productos distribuidos entre las diferentes categorías.

8 Para un análisis más profundo sobre dicho conflicto puede consultarse Varela \& Lotito (2009) y Cambiasso (2015).

9 En detalle, año por año: 1980: ROYAL; 1990: SUCHARD, MILKA; 1992: TANG, CLIGHT; 1994: TERRABUSI; 1996: MAYCO, CAPRI y VIZZOLINI; 1999: CANALE y TOSTI; 2001: se consolida como una compañía; 2007: se 
consolida como una empresa independiente; en 2008 Kraft adquiere el mercado de Galletitas perteneciente a Danone, en 2010 adquiere Cadbury.

10 Juan Grigera (2012) utiliza este concepto para analizar las transformaciones del sector industrial que tuvieron lugar en Argentina a partir de la década de 1970. En oposición a las lecturas que parten de la noción de desindustrialización, el autor sostiene que "el importante proceso de transformación de la economía mundial y las reacciones de Argentina por adaptarse a éstas se pueden captar con el mayor poder explicativo del concepto de reestructuración capitalista. Siguiendo esta idea se explica y comprende el desigual crecimiento de las ramas (es decir, desguace de algunas y promoción de otras), el aumento sostenido y pronunciado de la productividad (en algunas ramas con aumento del producto, en todos los casos con contracción del empleo), el proceso de concentración y centralización, y la profunda transformación de los procesos de trabajo (por ejemplo, desverticalización)" (p. 238).

11 En relación al incremento del trabajo asalariado durante la posconvertibilidad, es importante tener en cuenta que el empleo no registrado mantuvo porcentajes altos. Luego de una fuerte caída entre los años 2003 y 2007, la reducción del trabajo no registrado se estancó en niveles altos, dado que no se pasó el piso de los registros de la década de 1990 (ODS, 2014). A su vez, la falta de registro no es la única forma de precarización e informalidad laboral. La tercerización, los falsos autónomos y las cooperativas fraudulentas también constituyen estrategias del capital para eliminar derechos. En este orden, un informe reciente de la Organización Internacional del Trabajo (OIT) sostiene que la informalidad laboral alcanza a un $46,8 \%$ de los trabajadores del país, incluyendo a los cuentapropistas que se encuentran por fuera del sistema de seguridad social. 